\title{
A Similar but Distinctive Pattern of Impaired Cortical Excitability in First-Episode Schizophrenia and ADHD
}

\author{
Alkomiet Hasan $^{a, b}$ Marc Schneider ${ }^{c, d}$ Thomas Schneider-Axmann ${ }^{a}$ \\ Diane Ruge $^{\mathrm{e}}$ Wolfgang Retz ${ }^{c}$ Michael Rösler ${ }^{c}$ Peter Falkai ${ }^{b}$ \\ Thomas Wobrock ${ }^{\mathrm{a}}$
}

${ }^{a}$ Department of Psychiatry and Psychotherapy, Georg August University, Göttingen, ${ }^{b}$ Department of Psychiatry and Psychotherapy, Ludwig Maximilian University, Munich, and 'Institute of Forensic Psychology and Psychiatry,

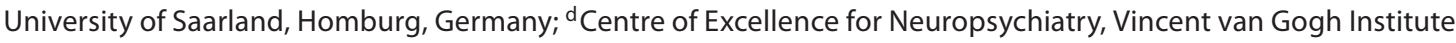

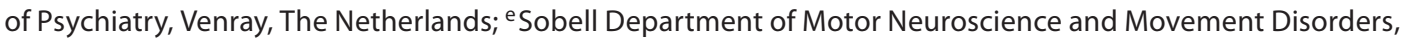
UCL Institute of Neurology, London, UK

\section{Key Words}

First-episode schizophrenia - Attention deficit hyperactivity disorder · Transcranial magnetic stimulation - Cortical excitability · Dopamine

\begin{abstract}
Background: First-episode schizophrenia (FE-SZ) and attention deficit hyperactivity disorder (ADHD) are both neuropsychiatric disorders associated with an impaired dopaminergic transmission. Though displaying different clinical phenotypes, a common pathophysiological pathway is discussed controversially. Several studies using transcranial magnetic stimulation (TMS) revealed abnormalities in human motor cortex excitability in both schizophrenia and ADHD patients. Studies on cortical excitability comparing these two diseases directly are lacking. Method: In this study, a total of 94 subjects were analyzed. Twenty-five FE-SZ patients were directly compared with 28 ADHD patients and 41 healthy controls $(\mathrm{HC})$. We investigated cortical excitability (inhibitory and facilitatory networks) with single- and pairedpulse TMS to the left and right motor cortex. Results: Compared to HC, FE-SZ/ADHD patients displayed an impaired
\end{abstract}

\section{KARGER}

Fax +4161306 1234

E-Mail karger@karger.ch

www.karger.com
(C) 2013 S. Karger AG, Basel

0302-282X/13/0672-0074\$38.00/0

Accessible online at:

www.karger.com/nps cortical inhibition over the left hemisphere. Apart from an enhanced intracortical facilitation, FE-SZ patients did not differ compared to ADHD patients in the main outcome measures. Both patient groups presented a dysfunctional hemispheric pattern of cortical inhibition and facilitation in comparison with HC. Conclusion: The results of this study indicate a pattern of cortical disinhibition and abnormal hemispheric balance of intracortical excitability networks in two different psychiatric diseases. These effects might be associated with an imbalance in GABAergic and dopaminergic transmission and might provide evidence for a common pathophysiological pathway of both diseases.

Copyright @ 2013 S. Karger AG, Basel

\section{Introduction}

Attention deficit hyperactivity disorder (ADHD) and schizophrenia are both heterogeneous disorders sharing a psychopathological profile of attention deficits and dif-

\section{A.H. and M.S. contributed equally to the study.}

Dr. Marc Schneider

Centre of Excellence for Neuropsychiatry, Vincent van Gogh Institute of Psychiatry Stationsweg 46

NL-5803 AC Venray (The Netherlands)

E-Mail MSchneider@vvgi.nl 
ficulties in social interaction. However, very clear distinguishing features between these diseases are delusions, hallucinations and negative symptoms as a clinical manifestation in schizophrenia patients [1]. There is comorbidity between these diseases and retrospective studies of individuals with schizophrenia have shown a history of premorbid or concomitant ADHD [2].

With regard to the pathophysiology, both conditions are associated with a dysfunctional dopaminergic transmission of the limbic-frontal network and other midbrain structures [3, 4]. An enhanced dopaminergic transmission in mesolimbic structures is associated with positive symptoms, whereas a dopaminergic underactivity in these brain structures has been linked to negative symptoms and cognitive dysfunction in schizophrenia patients. In ADHD, the pathophysiological role of the dopamine system remains elusive. Both, high midbrain dopamine activity or reduced dopamine activity are discussed to be involved in the appearance of ADHD symptoms, and the discussion about hypo- or hyperdopaminergic transmission imbalance remains contradictory $[4$, 5]. In some studies, a dopaminergic hyperfunction in the mesolimbic structures was correlated with hyperactivity and impulsivity and a dopaminergic hypofunction was found to underlie different cognitive symptoms [3, 4, 6-8]. Apart from dopamine, other neurotransmitter systems, especially GABAergic interneurons, are involved in the pathophysiology of these diseases $[9,10]$.

Using paired-pulse transcranial magnetic stimulation (TMS), a reduced cortical inhibition as another common pathophysiological state was found in both diseases [11, 12]. Paired-pulse TMS offers the possibility to investigate different mechanisms of cortical inhibition (short-interval intracortical inhibition, SICI) and of cortical facilitation (intracortical facilitation, ICF) in the human brain [13]. SICI is discussed to be mediated by GABAergic interneurons via $\mathrm{GABA}_{\mathrm{A}}$ receptors and ICF might result from primarily glutamatergic neurotransmission [14]. Furthermore, dopaminergic neurotransmission has an impact on SICI and ICF, because dopamine agonists lead to an enhancement of SICI and ICF and dopamine antagonists reduce SICI and ICF in healthy subjects [14]. The contralateral silent period (CSP), a single-pulse TMS measure, is at least in part the result of $\mathrm{GABA}_{\mathrm{B}}$-mediated inhibitory mechanisms $[15,16]$.

In schizophrenia patients, several TMS studies revealed abnormalities in motor-cortical excitability which have been linked to GABAergic and dopaminergic dysfunction [17-20]. Our research group has recently demonstrated a reduced SICI and a prolonged CSP in first-

TMS in First-Episode Schizophrenia and ADHD episode schizophrenia (FE-SZ). We hypothesized that reduced SICI points towards a $\mathrm{GABA}_{\mathrm{A}}$ deficit and the prolonged CSP may reflect compensatory increased $\mathrm{GABA}_{\mathrm{B}}$ transmission induced by hyperactivity of the dopaminergic system [11, 21].

Studies with child and adult ADHD patients displayed controversial results. Three different studies found abnormal SICI and ICF in children with ADHD, which normalized after administration of a psychostimulant [22-24]. Furthermore, a reduced duration of the CSP and the ipsilateral silent period, a measure of interhemispheric connectivity, is a common finding in children with $\mathrm{ADHD}$ $[23,24]$. In adults with ADHD, two studies revealed a reduced SICI $[12,25]$ and in another study the reduced SICI did not reach statistical significance [26]. Methodological differences in measuring and analyzing cortical excitability in adults with ADHD might be related to the negative finding of the latter study. The shift of ADHD symptoms during lifetime might be accompanied by adaptations in motor cortex excitability in some ADHD phenotypes. Furthermore, the higher variability of motor evoked potentials (MEPs) in ADHD might be the reason for the weak statistical significances of differences in cortical excitability between some ADHD adults and healthy controls (HC) [26]. As shown for ADHD in childhood, one study found a shortened ipsilateral silent period in adult ADHD, but there were no differences in CSP [27].

The present study was designed to directly compare measures of cortical excitability between ADHD and FESZ. To shed more light on the impact of cortical excitability on the underlying pathophysiology, $25 \mathrm{FE}$-SZ patients with minimal exposure to antipsychotics were compared with 28 adult ADHD patients with no history of psychotic or other axis I or II disorders and $41 \mathrm{HC}$. Therefore, previously and independently published samples (see Materials and Methods) were merged and newly analyzed regarding excitability differences between schizophrenia and ADHD patients. TMS over both hemispheres was used to test parameters of cortical excitability and functional hemispheric balance (SICI, ICF, CSP and motor thresholds).

Our aim was to focus on TMS measures on transmission mechanisms related to $\mathrm{GABA}_{\mathrm{A}}, \mathrm{GABA}_{\mathrm{B}}$ and glutamatergic systems in order to test the hypotheses of common pathophysiological motor networks in schizophrenia and ADHD. From the background outlined above, we hypothesized that schizophrenia patients would show the most pronounced abnormality in excitability that is less evident in ADHD. Another aim was to investigate functional hemispheric symmetry/connectivity using mea- 
sures of cortical excitability. Our hypothesis was that there is an imbalance in both disorders and schizophrenia patients would show the most pronounced deficit.

\section{Materials and Methods}

\section{Subjects}

The study sample consisted of 94 subjects. Twenty-eight patients with ADHD and 25 patients with FE-SZ (all paranoid subtype) from the same geographical area were recruited from inpatient and outpatient units and were compared with 41 healthy subjects. Parts of the sample have previously been published independently $[11,21,25]$, but this study provides new data on direct comparison of disorders, CSP in ADHD and late-ICF in FE-SZ. Subjects with a history of dementia, neurological illnesses and severe brain injuries were excluded from the study. Only righthanded subjects according to a standardized test of hand preference [28] were analyzed for this study.

A clinical psychiatrist, blinded to the aims of the study, and a member of the study group (T.W. or M.S.) made a consensus diagnosis according to the German version of the Structured Clinical Interview for DSM-IV [29]. Each subject underwent an assessment of disease severity (Clinical Global Impressions) [30] and an assessment of social functioning (Global Assessment of Functioning) [31]. In schizophrenia patients, an assessment of psychopathology (Positive and Negative Syndrome Scale) [32], the duration of illness, counted from the beginning of initial prodromal symptoms, and the duration of psychosis, counted from the onset of diagnostic/characteristic positive symptoms, were evaluated according to the foregoing publication [11]. All schizophrenia patients were treated with second-generation antipsychotics, but at the time of TMS measurements, no patient had been treated longer than 6 weeks continuously. We calculated chlorpromazine equivalents [33] for the cumulative and daily doses of the different antipsychotics to explore the influence of this medication on the study results.

The ADHD patients (combined type) were diagnosed according to the diagnostic criteria of DSM-IV (ADHD diagnostic checklist [34]), and had used no stimulant medication before. The Wender Utah Self-Rating Scale (short version) [35] was used to assess childhood ADHD symptoms. Patients with a clinical diagnosis of ADHD were included in the study if the Wender Utah Self-Rating Scale (short version) score was at least 30, and if at least 6 of 9 items of inattention and hyperactivity/impulsivity were present. As indicators of severity of symptoms, the ADHD selfrating scale for adults according to DSM-IV [34] was used (total score: 0-54; attention deficit and hyperactivity/impulsivity subscores: each maximal 27). The Structured Clinical Interview for DSM-IV Axis I and II Disorders was used to exclude further axis I and II DSM categorical diagnoses.

After a complete description of the study, written informed consent was obtained from each subject. The local ethics committee approved the protocol, which is in accordance with the Declaration of Helsinki.

\section{TMS Procedure}

As described previously [11], subjects were seated in a comfortable reclining chair with their arms supported passively. Electromyographic (EMG) recordings from the right and left first dorsal interosseous muscle (FDI) were made with surface electrodes. Raw signals were amplified, bandpass filtered (2 Hz to $10 \mathrm{kHz}$ ) and digitized using a commercial amplifier (Keypoint portable, Medtronic Co., Denmark). Each EMG recording was manually analyzed off-line. TMS was performed by using a MagPro $\times 100$ magnetic stimulator (Medtronic Co., Denmark) and focal TMS was applied to the hand area of the left and right motor cortex with a standard figure-of-eight magnetic coil. The optimal coil position was defined as the stimulation site that produced the largest MEP at moderately suprathreshold stimulation intensities (i.e. intensities that induce MEPs of about $0.5-1.5 \mathrm{mV}$ ) in the resting right and left FDI. The optimal position was marked to ensure that the coil was held in the correct position throughout the experiment. The coil was held tangentially to the head, with the handle pointing backwards and in an angle of $45^{\circ}$ lateral to the midline. This ensured that the induced current pointed forward and perpendicular to the central sulcus, which is optimal for producing transsynaptic activation of corticospinal neurons. The resting motor threshold (RMT), expressed as a percentage of maximum stimulator output, was defined as the lowest intensity that produced an MEP of $>50 \mu \mathrm{V}$ in 5 out of 10 trials in the relaxed FDI.

In accordance with standard TMS publications, SICI and ICF were obtained $[13,18]$, setting the intensity of the conditioning stimulus at $80 \%$ of the RMT and the test stimulus at an intensity that produced MEPs averaging $0.5-1.5 \mathrm{mV}$ in the resting FDI. SICI/ICF were measured with interstimulus intervals (ISIs) of 3, 5,7 and $15 \mathrm{~ms}$, and we performed a minimum of 8 trials with each ISI and 10 trials with the test stimulus alone. The effect of the conditioning stimulus on MEP amplitude of the test stimulus was determined as the ratio of the average amplitude of the conditioned MEP to the average amplitude of the unconditioned test MEP.

CSP duration was obtained in moderately tonically active FDI (25-30\% of maximal contraction) by stimulating the contralateral motor cortex with intensities of 120 and $140 \%$ of RMT. For each intensity, 8 trials were performed and the mean CSP duration calculated. The CSP duration was defined as the time from the MEP onset to the return of any voluntary EMG activity (absolute CSP) [36].

All measurements and clinical characterizations were performed by an experienced investigator (FE-SZ: T.W.; ADHD: M.S.), controlled by another experienced investigator (FE-SZ: M.S.; ADHD: T.W.), and corrected for outliers and extreme values. The data analysis was performed by A.H., T.W., M.S. and one statistician (T.S.A.).

\section{Statistical Analyses}

For statistical analyses, SPSS for Windows 17.0 was used. All tests were two-tailed. The level of significance was set at $\alpha=0.05$. Data are presented as mean \pm standard deviation unless otherwise indicated. Kolmogorov-Smirnov tests were applied to test normal distribution and logarithmic transformation was used if normality assumption was violated (SICI and ICF). Independent factor was group (FE-SZ, ADHD, HC) and dependent variables were RMT, ISI (3, 5, 7, $15 \mathrm{~ms}$ ) and CSP (intensities 120 and 140\% of RMT) on both hemispheres. As initial analyses, Pearson correlations between age and dependent variables were calculated and one-way ANOVA was performed to analyze if the factor gender influenced the dependent variables. If a significant influence 
Table 1. Demographic and clinical data of study subjects: interval-scaled data are presented as mean \pm standard deviation

\begin{tabular}{|c|c|c|c|c|c|}
\hline Variable & FE-SZ & ADHD & $\mathrm{HC}$ & Statistic & $\mathrm{p}$ \\
\hline Number & 25 & 28 & 41 & & \\
\hline Gender (M/F) & $18 / 7$ & $15 / 13$ & $20 / 21$ & $\chi^{2}(2)=3.522$ & 0.17 \\
\hline Age, years & $29.96 \pm 8.5$ & $32.36 \pm 9.1$ & $33.37 \pm 9.1$ & $\mathrm{~F}(2,91)=1.140$ & 0.32 \\
\hline \multicolumn{6}{|l|}{ Schizophrenia patients } \\
\hline PANSS positive & $21.96 \pm 5.7$ & - & - & & \\
\hline PANSS total & $92.00 \pm 14.5$ & - & - & & \\
\hline CGI & $5.88 \pm 0.6$ & - & - & & \\
\hline GAF & $30.0 \pm 10.5$ & - & - & & \\
\hline $\mathrm{CPZ1}$ & $356 \pm 204$ & - & - & & \\
\hline $\mathrm{CPZ2}$ & $7,565 \pm 7,887$ & - & - & & \\
\hline \multicolumn{6}{|l|}{ ADHD patients } \\
\hline
\end{tabular}

Values are expressed as $\chi^{2}$ statistics for categorical variables and F statistics for continuous variables. CGI = Clinical Global Impression; GAF = Global Assessment of Functioning; CPZ1 = chlorpromazine equivalents, daily dosage; CPZ2 = chlorpromazine equivalents, cumulative dosage; ADHD-SR = ADHD self-rating scale; $\mathrm{P}=$ error probability of first kind.

of these intervening variables was found, the main analyses were adjusted for these variables.

A repeated-measures multivariate analysis of variance (RMMANOVA) with the within-subject factor 'side' (left and right hemisphere) and the between-subject factor 'group' (HC, ADHD, FE-SZ) was initially performed. Only if this RM-MANOVA revealed a significant effect or a trend of the factor 'group' or 'side $\times$ group' interaction were separate ANOVAs for the left and right hemisphere performed. If appropriate, corrected Tukey's post hoc tests were performed to determine the differences between groups.

Pearson's product moment correlations between chlorpromazine equivalents and dependent variables were calculated for the FE-SZ patients.

\section{Results}

Sociodemographic and Clinical Characteristics, Influence of Intervening Variables Age and Sex on

Dependent Variables

There were no significant age and sex differences between groups. Schizophrenia patients presented a severe degree of illness and severe impairment of social functioning according to Clinical Global Impressions and Global Assessment of Functioning and moderate to severe positive and negative symptoms (Positive and Negative
Syndrome Scale). ADHD patients suffered from at least moderate symptoms according to the ADHD rating score.

All schizophrenia patients received an antipsychotic medication (but no other concomitant medication, like benzodiazepines) and the dosages of daily and cumulative antipsychotic medication (expressed as chlorpromazine equivalents) were $356.21 \pm 203.65$ (daily dosage) and 7,565.17 $\pm 7,886.79$ (cumulative dosage). ADHD patients and $\mathrm{HC}$ were unmedicated. For details of the sociodemographic and clinical characteristics, see table 1 .

Except for significant correlations between age and RMT (left: $\mathrm{r}=0.32$, d.f. $=92, \mathrm{p}=0.002$; right: $\mathrm{r}=0.31$, d.f. $=92, p=0.003)$, there was no significant influence of age on dependent variables. From one-way ANOVA, there was no significant difference between female and male subjects for any dependent variable.

\section{Resting Motor Threshold}

After adjustment for age, no statistically significant differences in RMT between groups were revealed (table 2).

\section{Intensity to Evoke MEPs of $1 \mathrm{mV}$}

There were no significant differences between groups (table 2). 
Table 2. Main outcome parameters

\begin{tabular}{|c|c|c|c|c|}
\hline Variable & FE-SZ & ADHD & $\mathrm{HC}$ & Statistics factor 'group' ( $\mathrm{p}$ values) \\
\hline RMT left & $47.68 \pm 6.55$ & $50.04 \pm 6.50$ & $47.49 \pm 5.93$ & \\
\hline RMT right & $49.00 \pm 7.43$ & $51.92 \pm 8.86$ & $48.29 \pm 8.92$ & - \\
\hline S1mV right & $59.50 \pm 10.33$ & $64.56 \pm 13.35$ & $63.65 \pm 14.48$ & - \\
\hline CSP120 left & $160.82 \pm 41.59$ & $164.72 \pm 33.78$ & $133.64 \pm 40.88$ & 0.013 \\
\hline CSP120 right & $165.04 \pm 55.03$ & $162.81 \pm 43.89$ & $166.48 \pm 37.38$ & 0.974 \\
\hline CSP140 right & $210.38 \pm 53.60$ & $197.40 \pm 57.13$ & $186.34 \pm 44.19$ & 0.303 \\
\hline SICI $3 \mathrm{~ms} \mathrm{left}$ & $39.61 \pm 29.97$ & $39.68 \pm 36.95$ & $23.90 \pm 17.63$ & 0.051 \\
\hline SICI 3 ms right & $51.11 \pm 57.97$ & $35.70 \pm 32.54$ & $32.00 \pm 22.99$ & 0.183 \\
\hline SICI $5 \mathrm{~ms}$ left & $82.88 \pm 83.18$ & $90.86 \pm 51.28$ & $63.86 \pm 35.86$ & - \\
\hline SICI 5 ms right & $86.87 \pm 74.57$ & $74.69 \pm 50.88$ & $81.48 \pm 55.73$ & - \\
\hline ICF $7 \mathrm{~ms}$ left & $110.68 \pm 110.01$ & $143.54 \pm 79.73$ & $94.35 \pm 49.14$ & 0.011 \\
\hline \multicolumn{3}{|c|}{$\begin{array}{l}\text { FE-SZ: First-episode schizophrenia, ADHD: attention deficit } \\
\text { hyperactivity disorder; HC: healthy controls; P: error probability } \\
\text { of first kind; RMT: resting motor threshold (in percent maximum } \\
\text { stimulator output); S1mV = intensity to evoke an MEP of } 1 \mathrm{mV} \\
\text { (peak to peak) in percent maximum stimulator output; CSP120 = } \\
\text { cortical contralateral silent period at } 120 \% \text { RMT (ms); CSP140 = } \\
\text { cortical contralateral silent period at } 140 \% \text { RMT (ms). SICI: Short- } \\
\text { interval intracortical inhibition at one certain interstimulus in- } \\
\text { terval; ICF: Intracortical facilitation at a defined interstimulus in- }\end{array}$} & \multicolumn{2}{|c|}{$\begin{array}{l}\text { terval. Data are presented as mean } \pm \text { standard deviation. Please } \\
\text { notice that statistical analyses on SICI and ICF values (percent } \\
\text { MEP amplitude with testpulse alone) were performed on log- } \\
\text { transformed values (raw values are presented in this table). Statis- } \\
\text { tics present results separately for the right and left hemisphere. - } \\
\text { RM-MANOVA did not show a side } \times \text { group interaction or a } \\
\text { group effect at least on trend level for this dependent variable, } \\
\text { therefore no further ANOVAs were performed separately for the } \\
\text { left and right hemisphere. }\end{array}$} \\
\hline
\end{tabular}

\section{Short-Interval Intracortical Inhibition and \\ Intracortical Facilitation}

The RM-MANOVA showed a significant 'side $\times$ group' interaction for ICF at $7 \mathrm{~ms}[\mathrm{~F}(2,86)=6.08, \mathrm{p}=$ $0.003]$ and for ICF at $15 \mathrm{~ms}[\mathrm{~F}(2,85)=5.20, \mathrm{p}=0.007]$ and a trend for the factor 'group' for SICI at $3 \mathrm{~ms}[\mathrm{~F}(2,86)=$ $3.04, \mathrm{p}=0.053]$.

The subsequent ANOVA showed separately for the left hemisphere a trend for the factor 'group' for SICI at $3 \mathrm{~ms}[\mathrm{~F}(2,90)=3.08, \mathrm{p}=0.051$, Tukey's post hoc test: trend for FE-SZ $<\mathrm{HC}(\mathrm{p}=0.080)]$ and a significant effect for ICF at $7 \mathrm{~ms}[\mathrm{~F}(2,90)=4.76, \mathrm{p}=0.011$, Tukey's post hoc test: ADHD > HC ( $\mathrm{p}=0.013)$, ADHD $>$ FE-SZ ( $\mathrm{p}=$ $0.044)$ ]. ANOVA for the right hemisphere revealed a trend for ICF at $15 \mathrm{~ms}[\mathrm{~F}(2,85)=2.92, \mathrm{p}=0.060$, Tukey's post hoc test: trend for ADHD < FE-SZ (p = 0.051)] (fig. 1, 2; table 2).

\section{Contralateral Silent Period}

For CSP120, RM-MANOVA revealed a significant effect for the factor 'group' $[\mathrm{F}(2,47)=3.84, \mathrm{p}=0.028]$ and a significant 'side $\times$ group' interaction $[\mathrm{F}(2,47)=6.22$, $\mathrm{p}=0.004]$. For CSP140, RM-MANOVA only showed a significant effect for the factor 'group' $[\mathrm{F}(2,46)=3.36$, $\mathrm{p}=0.043$ ], but no significant 'side $\times$ group' interaction $[\mathrm{F}(2,46)=0.17, \mathrm{p}=0.84]$.

ANOVA, considered separately for the left and right hemisphere, showed a significant effect for the factor 'group' on the left hemisphere for $\operatorname{CSP} 120[\mathrm{~F}(2,67)=4.61$, $\mathrm{p}=0.013$, Tukey's post hoc tests: ADHD $>\mathrm{HC}(\mathrm{p}=0.028)$, FE-SZ $>$ HC $(\mathrm{p}=0.042)]$ (fig. 3$)$ and CSP140 $[\mathrm{F}(2,63)=$ $3.47, \mathrm{p}=0.037$, Tukey's post hoc tests: FE-SZ $>\mathrm{HC}(\mathrm{p}=$ $0.032)$, but no difference on the right hemisphere for CSP120 $[\mathrm{F}(2,57)=0.03, \mathrm{p}=0.97]$ and CSP140 $[\mathrm{F}(2,55)=$ $1.22, \mathrm{p}=0.30]$. For details, see table 2 .

\section{Influence of Antipsychotic Medication on TMS \\ Parameters}

In the group of FE-SZ patients, we did not find a significant correlation between antipsychotic medication and TMS parameters. 


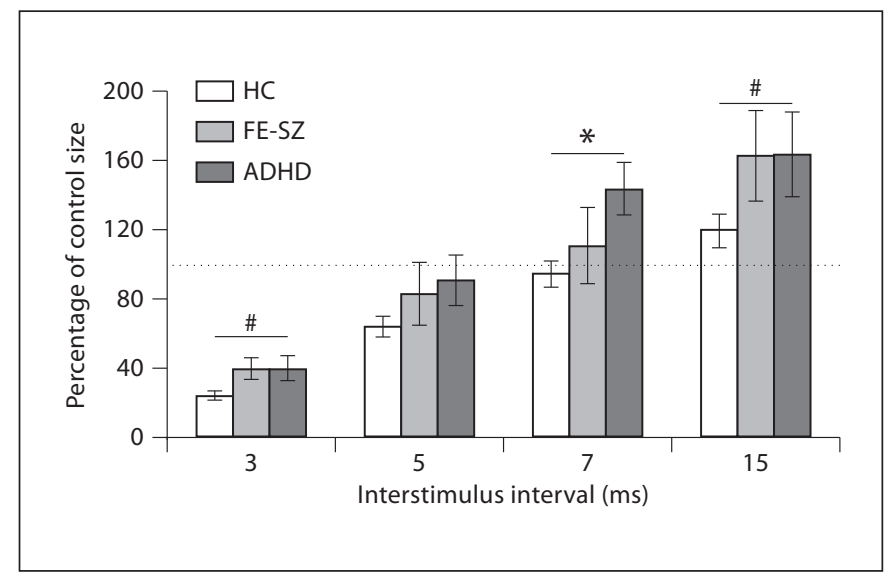

Fig. 1. Relative values for paired-pulse (SICI, ICF) measures on the left hemisphere expressed as percentage change compared to the test pulse in all groups. ${ }^{*} \mathrm{p}<0.05$ (RM-MANOVA), ${ }^{*} 0.05 \leq \mathrm{p} \leq$ 0.060 . At $3 \mathrm{~ms}, \mathrm{FE}-\mathrm{SZ}$ patients $(\mathrm{p}=0.080)$ had a trend towards a reduced SICI compared to HC. ADHD patients displayed an enhanced ICF at $7 \mathrm{~ms}$ compared to HC ( $\mathrm{p}=0.013)$ and to FE-SZ $(\mathrm{p}=0.044)$. The numeric differences for ISI $5 \mathrm{~ms}$ and ISI $15 \mathrm{~ms}$ did not reach significance. Data are presented as mean \pm standard error of the mean. $\mathrm{p}$ values are corrected in accordance to Tukey's test. Analyses were performed on logarithmic transformed data (see Materials and Methods).

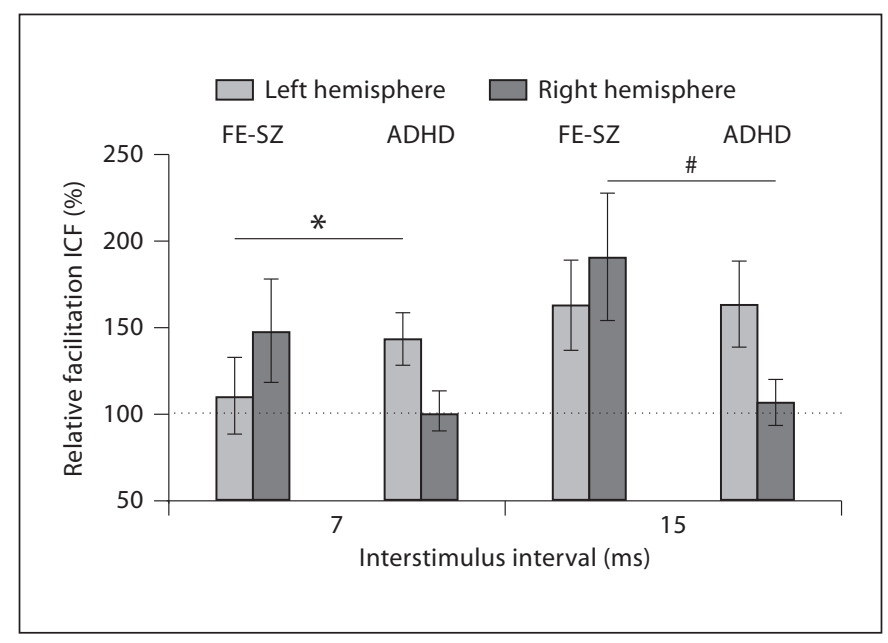

Fig. 2. Hemispheric differences of ICF at 7 and $15 \mathrm{~ms}$ in the FE-SZ and ADHD groups. At $7 \mathrm{~ms}$ on the left hemisphere, FE-SZ patients had a reduced ICF compared to ADHD patients $(p=0.044)$. At $15 \mathrm{~ms}$ on the right hemisphere, FE-SZ patients had an enhanced ICF compared to ADHD patients $(\mathrm{p}=0.051)$. Analyses were performed on logarithmic transformed data (see Materials and Methods). ${ }^{*} \mathrm{p}<0.05$ (RM-MANOVA), ${ }^{*} 0.050 \leq \mathrm{p} \leq 0.060$.

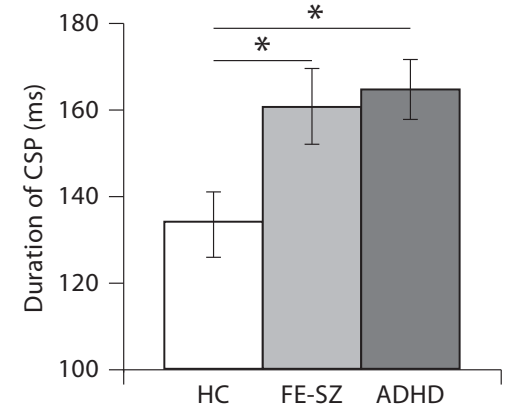

Fig. 3. CSP120 duration on the left hemisphere in all groups. $\operatorname{ADHD}(\mathrm{p}=0.028)$ and FE-SZ patients $(\mathrm{p}=0.042)$ present a longer CSP120 duration compared to HC. ADHD and FE-SZ patients do not differ concerning the CSP120 duration on the left hemisphere $(p=0.950)$. Data are presented as mean \pm standard error of the mean. $\mathrm{p}$ values are corrected in accordance to Tukey's test. ${ }^{*} \mathrm{p}<0.05$.

\section{Discussion}

Our main results are that both, FE-SZ and ADHD patients demonstrate a prolonged CSP and a cortical disinhibition measured by TMS protocols compared to HC. Direct statistical comparison showed that both patient groups have a similarly prolonged CSP compared to HC. Furthermore, FE-SZ and ADHD patients displayed a trend towards a reduced SICI in comparison to HC. A generally enhanced ICF was found in ADHD patients in the left hemisphere when compared to HC. Apart from an enhanced ICF at $15 \mathrm{~ms}$ over the right hemisphere and reduced ICF at $7 \mathrm{~ms}$ over the left hemisphere, TMS measures in FE-SZ patients did not significantly differ from ADHD patients.

Analysis of hemispheric effects revealed significant differences between groups. Both patient groups had a significantly different pattern of the hemispheric distribution of the inhibitory CSP and facilitatory ICF compared to HC. Interestingly, despite having a largely similar pattern of cortical disinhibition (CSP), a distinct hemispheric distribution of some facilitatory parameters could be detected between FE-SZ and ADHD patients (fig. 2).

The modified cortical excitability in both ADHD and FE-SZ patients compared to $\mathrm{HC}$ and the divergent hemispheric excitability pattern in both diseases are to our knowledge new and important findings that support the idea of a common pathological pathway in brain trans- 
mission in these diseases and raise questions about functional brain asymmetry in these diseases. In order to better understand the pattern of our result, some hypothetical and mechanistic explanations are provided below.

\section{General Remarks}

The role of SICI and CSP in schizophrenia remains controversial. Different studies pointed towards a reduced SICI in medicated chronic schizophrenia patients $[20,37,38]$ and FE-SZ patients [11], whereas other studies failed to show this difference $[18,19]$. CSP in medicated patients was found to be shortened [20] or prolonged [19, $21,39,40$ ] compared to $\mathrm{HC}$. The situation for ADHD is more clearly laid out. Two studies revealed a reduced SICI in adult ADHD patients [12, 25], whereas in another study this could not reach statistical significance [26]. In contrast to schizophrenia patients, ADHD patients did not show a prolonged CSP compared to HC in previous studies [27].

The reduced SICI and the enhanced ICF in FE-SZ and ADHD patients in our study can be considered as parameters of cortical disinhibition and might be linked to a dysfunction of the $\mathrm{GABA}_{\mathrm{A}}$ neurotransmission. The prolonged CSP in both patient groups can be best explained by an enhanced $\mathrm{GABA}_{\mathrm{B}}$ transmission [14]. To understand the relationship of different inhibitory and facilitatory pathways, the two following aspects are important.

\section{Possible Mode of Action with Regard to}

Intrahemispheric and Interhemispheric Networks

First, it was shown that CSP and SICI have an inverse relationship and that SICI is suppressed and ICF is facilitated during CSP. SICI seems to be controlled by presynaptic $\mathrm{GABA}_{B}$ receptor-mediated autoinhibition on inhibitory $\mathrm{GABA}_{\mathrm{A}}$ interneurons, similar to a presynaptic autoinhibition revealed by paired intracellular recordings in slices of rat and human motor cortex $[41,42]$. Therefore, one explanation of our results might be that the enhanced $\mathrm{GABA}_{\mathrm{B}}$-related inhibition (CSP) and the reduced $\mathrm{GABA}_{\mathrm{A}}$-related inhibition (SICI)/enhanced glutamaterelated facilitation (ICF) on the left hemisphere are interdependent and might reflect a compensatory circuit within this hemisphere [21]. However, we are not able to determine which network is dysfunctional and which network might compensate for this dysfunctionality.

Second, ADHD and FE-SZ patients presented a modified hemispheric pattern for CSP and ICF compared to HC. This is of particular importance, because abnormal brain laterality is a well-known finding in these diseases. Especially an atypical lateralization to the right hemi- sphere explored in EEG and structural MRI studies is an important finding in ADHD and schizophrenia [43-46]. It could be hypothesized that this relationship is linked to an impaired interhemispheric connectivity/inhibition. We did not investigate this in our sample. However, one study found an impaired interhemispheric inhibition in adult ADHD patients [27] and a reduced interhemispheric inhibition was observed in schizophrenia patients [47].

But why do FE-SZ patients differ from ADHD patients concerning the hemispheric distribution of ICF? We know from other studies that a cortical disinhibition of the left hemisphere (hyperexcitability) can result in a reduced facilitation (hypoexcitability) over the contralateral right hemisphere [48-50]. To understand our findings, the cortical disinhibition in both diseases on the left hemisphere (enhanced ICF in ADHD, reduced SICI in FE-SZ compared to HC) should be primarily taken into consideration. Therefore, we would like to hypothesize that the relatively reduced ICF in ADHD patients on the right hemisphere might compensate for the enhanced ICF on the left hemisphere. In FE-SZ, this compensatory mechanisms might be impaired and this might be reflected by the relatively facilitated ICF on the right hemisphere.

In general, we would like to discuss an intrahemispheric compensatory mechanism (reflected by CSP enhancement), which is unaffected in both patient groups, and an interhemispheric compensatory circuit (reflected by a reduction of ICF), which seems to be exclusively active in the ADHD group.

\section{Is There a Hypothetical Common Pathophysiological Pathway?}

It is a remarkable finding that FE-SZ and ADHD did not differ in our outcome measures, except for the aforementioned difference in ICF in both hemispheres. As hypothesized in the introduction, these diseases might in part share a common pathophysiological pathway and one possible pathway could be associated with dysfunctional dopaminergic transmission. Drug studies in healthy subjects and research on a hypodopaminergic disease (Parkinson's disease) revealed interesting findings about the effect of dopamine on TMS parameters. The CSP was prolonged after administration of dopamine agonists (L-DOPA, pergolide) and shortened in patients with Parkinson's disease [14, 51]. Hence, the prolonged duration of the CSP over the left hemisphere might be linked with the theory of dopaminergic hyperactivity in schizophrenia and ADHD. Here, the enhanced dopaminergic input would lead to a compensatory increase of GABAergic transmission and probably cause the CSP 
prolongation [52]. In ADHD, there is a discussion about the possibility of dopaminergic dysbalance in frontal subcortical regions. Recently, we demonstrated an improvement of SICI in ADHD with long-acting methylphenidate [53]. This was interpreted as a dopamine-related response to the stimulants, possibly reflecting a complex dopaminergic-GABAergic interaction. However, with the TMS technique we are not able to determine the localization of the affected brain regions involved in dopaminergic transmission nor directly probe dopaminergic functions. Therefore, currently this theory remains speculative.

\section{Limitations}

Our comparative study has several limitations. First of all, all schizophrenia patients received antipsychotic medication, which might have an impact on the results of TMS paradigms. We did not find a correlation between chlorpromazine equivalents on the results of the TMS measures and an influence of antipsychotic drugs on TMS measurements is discussed controversially. One recent study showed that unmedicated schizophrenia patients and patients treated with second-generation antipsychotics (risperidone, olanzapine, quetiapine) had a reduced CSP and that patients treated with clozapine had significantly less SICI and a longer CSP compared to HC [37]. All of our patients received second-generation antipsychotic medication but, in contrast to Liu et al. [37], none of our patients received clozapine. Also, it is important to recognize that the schizophrenia patients in our study were at their first episode with a relatively short treatment history whereas Liu and co-authors investigated chronically ill schizophrenia patients and patients with a schizoaffective disorder. However, reviewing the literature, we would expect that antipsychotics should compensate for the reduced inhibition and not enhance it $[11,14,21]$. Furthermore, it should be considered that the drug-naïve adult ADHD patients showed a motor disinhibition which was not present in HC. Finally, there were no significant differences between ADHD and FESZ patients with regard to inhibitory TMS parameters. This might support the hypothesis that diminished cortical inhibition is genuine to these diseases. Nevertheless, our results in adult ADHD patients are partially in contrast with the results of two previous studies, where significant differences in SICI and CSP could not be found $[26,27]$. This could be due to different methodical procedures in TMS techniques as well as to divergent recruitment strategies. However, our results stay well in line with the diminished SICI seen in children and adolescents with ADHD $[22,54]$.

TMS in First-Episode Schizophrenia and ADHD
Although FE-SZ and ADHD patients present a similar cortical disinhibition, we cannot rule out that different mechanisms may underlie these effects and that these are not specifically restricted to these diseases [55]. Cortical disinhibition, as revealed by TMS, might reflect a general characteristic of psychiatric conditions, because different other psychiatric diseases, like depression or obsessivecompulsive disorder, are known to show a cortical disinhibition [56, 57].

ADHD is a dimensional diagnosis, but diagnosis of schizophrenia is based on the qualitative dimensions of symptoms. However, they still share some common executive dysfunctions (e.g. working memory, impulsivity) and attention deficits, whereas the motor symptoms usually are opposite. Despite the different subsumed pathophysiology and phenotypes, a common dopaminergic pathway has been discussed in both diseases $[3,4]$. However, it should be considered that dopamine has a neuromodulatory function. The dopamine effect depends on spontaneous neuronal activity, dopamine concentration and dopaminergic subreceptors [55]. Therefore, the transfer of our TMS findings to the complex aspects of dopaminergic transmission mechanisms to both diseases can be done only in a limited manner. Thus, the interpretation of our TMS results should be mainly focused on the discussed primary GABAergic transmission pathways.

Finally, it should be noted that overlapping parts of this study have been published before (see method section). However, this is the first study which directly compares cortical excitability in FE-SZ and ADHD. The FESZ resembling abnormal CSP has never been explored in ADHD and abnormal late ICF data $(15 \mathrm{~ms})$ for FE-SZ have never been characterized in previous studies. Further, our study reveals TMS-induced functional hemispheric asymmetry for both diagnostic groups. Previous papers from our groups provide detailed discussions for each diagnostic group with a specific focus on effects of one hemisphere [11, 21, 25].

In conclusion, the results of our study provide evidence for a cortical disinhibition in ADHD and FE-SZ and for a common pathophysiological pathway. Furthermore, we were able to present a functional hemispheric asymmetry in both diseases.

\section{Disclosure Statement}

The authors deny any potential conflict of interest regarding the subject of this report. 


\section{References}

$>1$ Ross RG, Olincy A, Harris JG, Sullivan B, Radant A: Smooth pursuit eye movements in schizophrenia and attentional dysfunction: adults with schizophrenia, ADHD, and a normal comparison group. Biol Psychiatry 2000;48:197-203.

-2 Marsh PJ, Williams LM: ADHD and schizophrenia phenomenology: visual scanpaths to emotional faces as a potential psychophysiological marker? Neurosci Biobehav Rev 2006;30:651-665.

$>3$ Pani L: Clinical implications of dopamine research in schizophrenia. Curr Med Res Opin 2002;18(suppl 3):s3-s7.

4 Solanto MV: Dopamine dysfunction in AD/ $\mathrm{HD}$ : integrating clinical and basic neuroscience research. Behav Brain Res 2002;130:6571.

5 Ohno M: The dopaminergic system in attention deficit/hyperactivity disorder. Congenit Anom (Kyoto) 2003;43:114-122.

$\checkmark 6$ Durston S: A review of the biological bases of ADHD: what have we learned from imaging studies? Ment Retard Dev Disabil Res Rev 2003;9:184-195.

$\checkmark 7$ Carriere P, Bonhomme D, Lemperiere T: Amisulpride has a superior benefit/risk profile to haloperidol in schizophrenia: results of a multicentre, double-blind study (the amisulpride study group). Eur Psychiatry 2000;15:321-329.

$>8$ Jucaite A, Fernell E, Halldin C, Forssberg H, Farde L: Reduced midbrain dopamine transporter binding in male adolescents with attention-deficit/hyperactivity disorder: association between striatal dopamine markers and motor hyperactivity. Biol Psychiatry 2005;57:229-238.

$\checkmark 9$ Pouget P, Wattiez N, Rivaud-Pechoux S, Gaymard B: A fragile balance: perturbation of GABA mediated circuit in prefrontal cortex generates high intraindividual performance variability. PLoS One 2009;4:e5208.

10 Hashimoto T, Bazmi HH, Mirnics K, Wu Q, Sampson AR, Lewis DA: Conserved regional patterns of GABA-related transcript expression in the neocortex of subjects with schizophrenia. Am J Psychiatry 2008;165:479-489.

- 11 Wobrock T, Schneider M, Kadovic D, Schneider-Axmann T, Ecker UK, Retz W, Rosler M, Falkai P: Reduced cortical inhibition in first-episode schizophrenia. Schizophr Res 2008;105:252-261.

-12 Richter MM, Ehlis AC, Jacob CP, Fallgatter AJ: Cortical excitability in adult patients with attention-deficit/hyperactivity disorder (ADHD). Neurosci Lett 2007;419:137141.

$\checkmark 13$ Kujirai T, Caramia MD, Rothwell JC, Day BL, Thompson PD, Ferbert A, Wroe S, Asselman P, Marsden CD: Corticocortical inhibition in human motor cortex. J Physiol 1993; 471:501-519.
4 Ziemann U: TMS and drugs. Clin Neurophysiol 2004; 115:1717-1729.

15 Werhahn KJ, Kunesch E, Noachtar S, Benecke R, Classen J: Differential effects on motorcortical inhibition induced by blockade of GABA uptake in humans. J Physiol 1999;517(Pt 2):591-597.

16 Siebner HR, Dressnandt J, Auer C, Conrad B: Continuous intrathecal baclofen infusions induced a marked increase of the transcranially evoked silent period in a patient with generalized dystonia. Muscle Nerve 1998;21: 1209-1212.

17 Pascual-Leone A, Manoach DS, Birnbaum R, Goff DC: Motor cortical excitability in schizophrenia. Biol Psychiatry 2002;52:2431.

18 Eichhammer P, Wiegand R, Kharraz A, Langguth B, Binder H, Hajak G: Cortical excitability in neuroleptic-naive first-episode schizophrenic patients. Schizophr Res 2004; 67:253-259.

19 Daskalakis ZJ, Christensen BK, Chen R, Fitzgerald PB, Zipursky RB, Kapur S: Evidence for impaired cortical inhibition in schizophrenia using transcranial magnetic stimulation. Arch Gen Psychiatry 2002;59: 347-354.

20 Fitzgerald PB, Brown TL, Daskalakis ZJ, Kulkarni J: A transcranial magnetic stimulation study of inhibitory deficits in the motor cortex in patients with schizophrenia. Psychiatry Res 2002;114:11-22.

21 Wobrock T, Schneider-Axmann T, Retz W, Rosler M, Kadovic D, Falkai P, Schneider M: Motor circuit abnormalities in first-episode schizophrenia assessed with transcranial magnetic stimulation. Pharmacopsychiatry 2009;42:194-201.

22 Moll GH, Heinrich H, Trott G, Wirth S, Rothenberger A: Deficient intracortical inhibition in drug-naive children with attention-deficit hyperactivity disorder is enhanced by methylphenidate. Neurosci Lett 2000;284:121-125.

23 Buchmann J, Gierow W, Weber S, Hoeppner J, Klauer T, Benecke R, Haessler F, Wolters A Restoration of disturbed intracortical motor inhibition and facilitation in attention deficit hyperactivity disorder children by methylphenidate. Biol Psychiatry 2007;62:963969.

24 Gilbert DL, Bansal AS, Sethuraman G, Sallee FR, Zhang J, Lipps T, Wassermann EM: Association of cortical disinhibition with tic, ADHD, and OCD severity in Tourette syndrome. Mov Disord 2004; 19:416-425.

25 Schneider M, Retz W, Freitag C, Irsch J, Graf P, Retz-Junginger P, Rosler M: Impaired cortical inhibition in adult ADHD patients: a study with transcranial magnetic stimulation. J Neural Transm Suppl 2007:303-309.
26 Hoeppner J, Neumeyer M, Wandschneider R, Herpertz SC, Gierow W, Haessler F, Buchmann J: Intracortical motor inhibition and facilitation in adults with attention deficit/ hyperactivity disorder. J Neural Transm 2008;115:1701-1707.

27 Hoeppner J, Wandschneider R, Neumeyer M, Gierow W, Haessler F, Herpertz SC, Buchmann J: Impaired transcallosally mediated motor inhibition in adults with attentiondeficit/hyperactivity disorder is modulated by methylphenidate. J Neural Transm 2008; 115:777-785.

28 Annett M: A classification of hand preference by association analysis. Br J Psychol 1970;61:303-321.

29 Wittchen HU, Wunderlich U, Gruschwitz S, Zaudig M: Strukturiertes klinisches Interview für DSM-IV; in Spitzer RL, Williams JB, Gibbon M, First MB (eds): Achse I: Psychische Störungen. A German, Advanced Adaptation of the Original SCID Version. Göttingen, Hogrefe, 1997.

30 National Institute of Mental Health: CGI: Clinical Global Impressions; in Guy W, Bonato RR (eds): Manual for the ECDEU Assessment Battery. Chevy Chase, National Institute of Mental Health, 1976.

31 Endicott J, Spitzer RL, Fleiss JL, Cohen J: The global assessment scale. A procedure for measuring overall severity of psychiatric disturbance. Arch Gen Psychiatry 1976;33:766-771.

-32 Kay SR, Fiszbein A, Opler LA: The positive and negative syndrome scale (PANSS) for schizophrenia. Schizophr Bull 1987;13:261276.

33 Woods SW: Chlorpromazine equivalent doses for the newer atypical antipsychotics. J Clin Psychiatry 2003;64:663-667.

34 Rösler M, Retz W, Retz-Junginger P, Thome J, Supprian T, Nissen T, Stieglitz RD, Blocher D, Hengesch G, Trott GE: Tools for the diagnosis of attention-deficit/hyperactivity disorder in adults. Self-rating behaviour questionnaire and diagnostic checklist. Nervenarzt 2004;75:888-895.

35 Wender PH, Wolf LE, Wasserstein J: Adults with ADHD. An overview. Ann NY Acad Sci 2001;931:1-16.

36 Daskalakis ZJ, Christensen BK, Chen R, Fitzgerald PB, Zipursky RB, Kapur S: Effect of antipsychotics on cortical inhibition using transcranial magnetic stimulation. Psychopharmacology (Berl) 2003;170:255-262.

-37 Liu SK, Fitzgerald PB, Daigle M, Chen R, Daskalakis ZJ: The relationship between cortical inhibition, antipsychotic treatment, and the symptoms of schizophrenia. Biol Psychiatry 2009;65:503-509.

>38 Hasan A, Nitsche MA, Rein B, SchneiderAxmann T, Guse B, Gruber O, Falkai P, Wobrock T: Dysfunctional long-term potentiation-like plasticity in schizophrenia revealed by transcranial direct current stimulation. Behav Brain Res 2011;224:15-22. 
39 Bajbouj M, Gallinat J, Niehaus L, Lang UE, Roricht S, Meyer BU: Abnormalities of inhibitory neuronal mechanisms in the motor cortex of patients with schizophrenia. Pharmacopsychiatry 2004;37:74-80.

40 Hasan A, Nitsche MA, Herrmann M Schneider-Axmann T, Marshall L, Gruber O, Falkai P, Wobrock T: Impaired long-term depression in schizophrenia: a cathodal tDCS pilot study. Brain Stimul 2012;5:475483 .

-41 McDonnell MN, Orekhov Y, Ziemann U: The role of GABA(b) receptors in intracortical inhibition in the human motor cortex. Exp Brain Res 2006;173:86-93.

-42 Ni Z, Gunraj C, Chen R: Short interval intracortical inhibition and facilitation during the silent period in human. J Physiol 2007; 583:971-982.

-43 Hale TS, Smalley SL, Walshaw PD, Hanada G, Macion J, McCracken JT, McGough JJ, Loo SK: Atypical EEG beta asymmetry in adults with ADHD. Neuropsychologia 2010; 48:3532-3539.

44 White T, Nelson M, Lim KO: Diffusion tensor imaging in psychiatric disorders. Top Magn Reson Imaging 2008;19:97-109.
45 Shapleske J, Rossell SL, Woodruff PW, David AS: The planum temporale: a systematic, quantitative review of its structural, functional and clinical significance. Brain Res Brain Res Rev 1999;29:26-49.

46 Schmitt A, Hasan A, Gruber O, Falkai P. Schizophrenia as a disorder of disconnectivity. Eur Arch Psychiatry Clin Neurosci 2011; 261(Suppl 2):S150-S154.

47 Fitzgerald PB, Brown TL, Daskalakis ZJ: The application of transcranial magnetic stimulation in psychiatry and neurosciences research. Acta Psychiatr Scand 2002;105:324340.

48 Suppa A, Ortu E, Zafar N, Deriu F, Paulus W, Berardelli A, Rothwell JC: Theta burst stimulation induces after-effects on contralateral primary motor cortex excitability in humans. J Physiol 2008;586:4489-4500.

49 Wassermann EM, Wedegaertner FR, Ziemann U, George MS, Chen R: Crossed reduction of human motor cortex excitability by $1-\mathrm{Hz}$ transcranial magnetic stimulation. Neurosci Lett 1998;250:141-144.

50 Heide G, Witte OW, Ziemann U: Physiology of modulation of motor cortex excitability by low-frequency suprathreshold repetitive transcranial magnetic stimulation. Exp Brain Res 2006;171:26-34.

51 Cantello R, Tarletti R, Civardi C: Transcranial magnetic stimulation and Parkinson's disease. Brain Res Brain Res Rev 2002;38: 309-327.
Beauregard M, Ferron A: Dopamine modulates the inhibition induced by GABA in rat cerebral cortex: an iontophoretic study. Eur J Pharmacol 1991;205:225-231.

53 Schneider M, Retz W, Rösler M: Effects of long-acting methylphenidate in adults with attention deficit hyperactivity disorder: a study with paired-pulse transcranial magnetic stimulation. Neuropsychobiology 2011; 64:195-201.

54 Moll GH, Heinrich H, Trott GE, Wirth S, Bock N, Rothenberger A: Children with comorbid attention-deficit-hyperactivity disorder and tic disorder: evidence for additive inhibitory deficits within the motor system. Ann Neurol 2001;49:393-396.

55 Nitsche MA, Monte-Silva K, Kuo MF, Paulus W: Dopaminergic impact on cortical excitability in humans. Rev Neurosci 2010;21: 289-298.

56 Bajbouj M, Lisanby SH, Lang UE, DankerHopfe H, Heuser I, Neu P: Evidence for impaired cortical inhibition in patients with unipolar major depression. Biol Psychiatry 2006;59:395-400.

57 Greenberg BD, Ziemann U, Cora-Locatelli G, Harmon A, Murphy DL, Keel JC, Wassermann EM: Altered cortical excitability in obsessive-compulsive disorder. Neurology 2000;54:142-147. 